\title{
Competition between quantum spin tunneling and Kondo effect
}

\author{
David Jacob ${ }^{1, a}$ and Joaquin Fernández-Rossier ${ }^{2,3}$ \\ 1 Max-Planck-Institut für Mikrostrukturphysik, Weinberg 2, 06120 Halle, Germany \\ 2 International Iberian Nanotechnology Laboratory (INL), 4715-330 Braga, Portugal \\ 3 Departamento de Física Aplicada, Universidad de Alicante, 03690 San Vicente del Raspeig, Spain
}

Received 28 June 2016 / Received in final form 21 July 2016

Published online 3 October 2016

(C) The Author(s) 2016. This article is published with open access at Springerlink.com

\begin{abstract}
Quantum spin tunneling and Kondo effect are two very different quantum phenomena that produce the same effect on quantized spins, namely, the quenching of their magnetization. However, the nature of this quenching is very different so that quantum spin tunneling and Kondo effect compete with each other. Importantly, both quantum spin tunneling and Kondo effect produce very characteristic features in the spectral function that can be measured by means of single spin scanning tunneling spectroscopy and allows to probe the crossover from one regime to the other. We model this crossover, and the resulting changes in transport, using a non-perturbative treatment of a generalized Anderson model including magnetic anisotropy that leads to quantum spin tunneling. We predict that, at zero magnetic field, integer spins can feature a split-Kondo peak driven by quantum spin tunneling.
\end{abstract}

\section{Introduction}

Quantum spin tunneling (QST) and Kondo effect are two ubiquitous and widely studied [1-3] phenomena in the broad field of nanoscale magnetism. They both turn a spin system with a doubly degenerate ground state into a system with a unique ground state with null magnetization. QST affects quantized integer spins with magnetic anisotropy, such as single molecule magnets [1], magnetic impurities in insulators [2] and magnetic adatoms [4,5] and molecules [6] on surfaces. The Kondo effect is most often associated with half-integer spins, but it has been observed in a variety of integer spin systems, such as quantum dots with an even number of electrons [7], various integer spin magnetic molecules [8-10] and molecular oxygen (spin $S=1$ ) adsorbed on gold [11].

The Kondo effect arises when a local spin is exchange coupled to itinerant electrons that respond dynamically to screen the magnetic moment of the impurity [3]. This dynamical response leads to a resonance in the local density of states at zero energy, that emerges as a zero bias Fano feature in the transport spectroscopy curves, $G(V)$, where $G \equiv \frac{d I}{d V}$. The observation of the Kondo resonance in individual magnetic atoms [12] and molecules [9] by means of Scanning Tunneling Microscope (STM) spectroscopy has been reported numerous times in the last two decades. The natural energy scale that characterizes the Kondo effect is roughly given by the width of this resonance which depends on the tunneling rate $\Gamma$ for elec-

\footnotetext{
${ }^{a}$ e-mail: djacob@mpi-halle.de
}

trons between the localized atomic orbitals and the extended states of the surface, and the charging energy of the atom, $\mathcal{U}_{N} \equiv E(N+1)-E(N)$.

QST can occur for integer spins with negative dominant uniaxial anisotropy $D<0$ : in this case the ground state of a spin $S$ is doubly degenerate and consists of the two states with opposite and maximal spin projection $m_{z}= \pm S$, which are separated by an energy barrier $\sim D S^{2}$. A finite in-plane magnetic anisotropy $E$ then allows quantum tunneling between the two spin states, lifting the degeneracy $[13,14]$ of the ground state by the tunnel splitting $\Delta_{0}$. Thus the ground state is a linear combination of two spin states with opposite spin projection and null magnetization $[15,16]$. The dynamical spin response function acquires a pole at $\hbar \omega=\Delta_{0}$ so that QST can be probed spectroscopically. Whereas in large spin molecular magnets $\Delta_{0}$ is so small that it can only be inferred indirectly [14], for systems with $S=1,2$, such as magnetic adatoms and small magnetic molecules deposited on conducting substrates, the spin excitations, and thereby the QST splittings, have been resolved directly by means of inelastic electron tunneling spectroscopy (IETS) using STM [4]. The corresponding $G(V)$ spectra show step features and, in general, no Kondo peak. It is customarily assumed that these excitation energies are a property of the atomic/molecular quantized spin, weakly dressed [4,16-18] by its Kondo exchange coupling.

Whether a given magnetic atom or molecule will show a stepwise $G(V)$ spectrum [4-6] or a zero bias Kondo resonance depends on the strength of the Kondo exchange $J$ which is controlled by the ratio $\Gamma / \mathcal{U}$. 
Thus, FePc molecules, that in vacuum have $S=1$, display a Kondo feature when deposited on $\mathrm{Au}$ (111) [9] and inelastic steps when deposited on oxidized $\mathrm{Cu}(110)$ [6]. Moreover, the joint observation of a zero bias Kondo resonance together with stepwise inelastic spin excitations has been reported for individual magnetic atoms $[17,19]$. Importantly, it is possible to devise experiments $[8,17,20]$ in which the Kondo interaction could be tuned, making it relevant to address the question of how the $G(V)$ spectra evolve from the weak to the strong coupling regime. The magnitude of the quantum spin tunneling splitting can also be modulated by application of a magnetic field along the hard axis direction $[13,14]$. In this work we address how the competition between Kondo screening and QST affect the STM inelastic conductance and we predict a new physical phenomenon, the splitting of the Kondo peak at zero magnetic field due to quantum spin tunneling.

Both the inelastic steps [21] and the Kondo features [22-25] can be described using a Kondo Hamiltonian where the atomic spin is described with a single-ion quantized spin interacting, via exchange, with the conduction electrons of the surface. In particular, the interplay between magnetic anisotropy and Kondo screening has been thoroughly studied using the Kondo model [17,18,22-25]. Here we present a more general approach, based on a nonperturbative treatment of a multi-orbital Anderson model for the adatom coupled to the substrate, that permits to include atomic charge fluctuations that are effectively frozen in the Kondo model. As we show below, valence fluctuations have a similar effect on the spectra as Kondo exchange. In addition, density functional theory (DFT) calculations show that often charge is not quantized in magnetic adatom systems [26,27].

\section{Model and method}

We consider tunneling between an STM tip and a magnetic adatom coupled to a surface as shown in Figure 1a. Assuming weak coupling to the STM tip (tunneling regime) [28] the low-bias conductance can be directly related to the adatom density of states (technically, the many-body spectral function) $\rho_{\alpha}(\omega)$,

$$
\mathcal{G}(V)=\frac{2 e^{2}}{\hbar} \sum_{\alpha} \Gamma_{\alpha}^{\mathrm{tip}} \rho_{\alpha}(\mathrm{eV})
$$

where $\Gamma_{\alpha}^{\text {tip }}=\pi\left|V_{\alpha}^{\text {tip }}\right|^{2} \rho_{\text {tip }}$ is the (weak) tunneling rate of electrons between the adatom orbitals $\alpha$ and the STM tip. We have assumed the DOS of the STM tip $\rho_{\text {tip }}$ is energy independent around the Fermi level. Note however, that in general different orbitals couple differently to the STM tip so that the contribution of the individual channels to the total conductance may differ. We neglect direct tunneling into surface states in equation (1). This is a good approximation when the magnetic atoms are separated from the metallic surface by a decoupling insulating layer, such as $\mathrm{Cu}_{2} \mathrm{~N} / \mathrm{Cu}(100)[4,17,19], \mathrm{CuO} / \mathrm{Cu}[6]$ and h-BN/Rh(111) [20]. This approximation does not capture

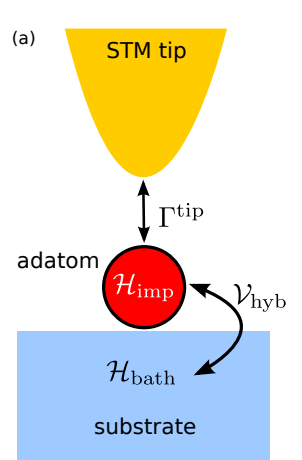

(b)

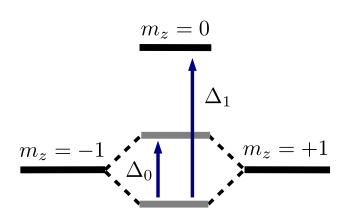

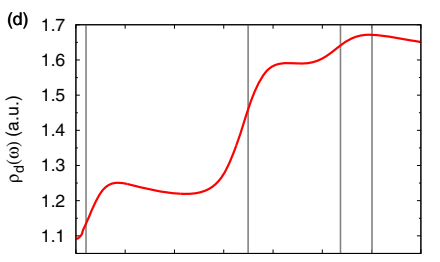

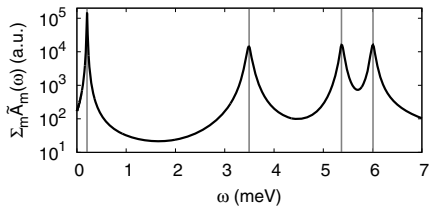

(c)

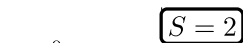

$\underline{m_{z}=0}$

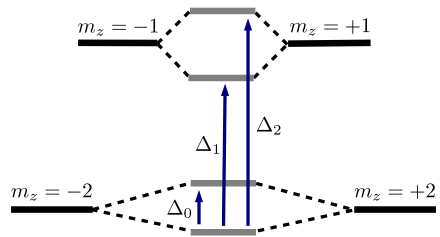

Fig. 1. (a) Schematic model of experimental setup for measuring excitation spectra of a magnetic adatom on a surface with STM tip. (b) Schematic energy spectrum of adatom GS multiplet for $S=1$ for negative uniaxial anisotropy $D<0$ and finite in-plane anisotropy $E$. (c) Same as (b) but for $S=2$. (d) Comparison of PP spectrum $\tilde{A}_{m}(\omega)=A_{m}(\omega) / f(-\omega)$ for the four lowest excitations (bottom) and the real electron spectrum (top) for $S=2, D=-1.55 \mathrm{meV}$ and $E=0.35 \mathrm{meV}$. The vertical grey lines indicate the positions of the PP energies $E_{m}^{*}$ w.r.t. the GS PP energy $E_{0}^{*}$.

the Fano interference effect relevant [12] when the tipatom channel interferes with the direct tip-surface tunneling path [29].

We describe the magnetic atom on the surface by a multi-orbital Anderson model,

$$
\mathcal{H}=\mathcal{H}_{\text {imp }}+\mathcal{H}_{\text {bath }}+\mathcal{V}_{\text {hyb }}
$$

where the Hamiltonian of the Anderson impurity site $\mathcal{H}_{\text {imp }}$ describes the strongly interacting $3 d$-levels that yield the spin of the magnetic atom, and includes a term that accounts for magnetic anisotropy:

$$
\begin{aligned}
\mathcal{H}_{\mathrm{imp}}= & \epsilon_{d} \hat{N}_{d}+\sum_{\alpha \sigma \neq \alpha^{\prime} \sigma^{\prime}} U \hat{n}_{\alpha \sigma} \hat{n}_{\alpha^{\prime} \sigma^{\prime}}-\sum_{\alpha \neq \alpha^{\prime}} J_{\mathrm{H}} \boldsymbol{S}_{\alpha} \cdot \boldsymbol{S}_{\alpha^{\prime}} \\
& +D \hat{S}_{z}^{2}+E\left(\hat{S}_{x}^{2}-\hat{S}_{y}^{2}\right) .
\end{aligned}
$$

$\epsilon_{d}$ are the single-particle energies of the $d$-levels, $\hat{N}_{d}=$ $\sum_{\alpha, \sigma} \hat{n}_{\alpha \sigma}$ is the number operator for all $d$-levels $\alpha=$ $1, \ldots, M, \hat{n}_{\alpha \sigma}=d_{\alpha \sigma}^{\dagger} d_{\alpha \sigma}$ is the number operator of an individual $d$-level $\alpha$ with spin $\sigma, U$ is the effective Coulomb repulsion, $J_{\mathrm{H}}$ the Hund's coupling, and $\boldsymbol{S}_{\alpha}$ measures the total spin of an individual $d$-level $\alpha$, i.e. $\boldsymbol{S}_{\alpha}=\sum_{\sigma \sigma^{\prime}} d_{\alpha \sigma}^{\dagger} \boldsymbol{\tau}_{\sigma \sigma^{\prime}} d_{\alpha \sigma^{\prime}}$. The crystal field splitting of the $d$-levels together with the spin-orbit coupling (SOC) gives rise [2] to magnetic anisotropy (MA) which in our simplified model is taken into account by the effective spin 
Hamiltonian given by the last term of (3) where $D$ is the uniaxial anisotropy and $E$ the in-plane anisotropy [1].

The second term in (2) describes the conduction electron bath in the surface:

$$
\mathcal{H}_{\text {bath }}=\sum_{k, \alpha, \sigma} \varepsilon_{k \alpha} c_{k \alpha \sigma}^{\dagger} c_{k \alpha \sigma} .
$$

The third term in (2) is the so-called hybridization term which describes the coupling between the impurity and the conduction electron bath:

$$
\mathcal{V}_{\text {hyb }}=\sum_{k, \alpha, \sigma} V_{k \alpha}\left(c_{k \alpha \sigma}^{\dagger} d_{\alpha \sigma}+d_{\alpha \sigma}^{\dagger} c_{k \alpha \sigma}\right) .
$$

Integrating out the bath degrees of freedom one obtains the so-called hybridization function:

$$
\Delta_{\alpha}^{\mathrm{hyb}}(\omega)=\sum_{k} \frac{\left|V_{k \alpha}\right|^{2}}{\omega+\mu-\varepsilon_{k \alpha}+i \eta} .
$$

Its (negative) imaginary part $\Gamma_{\alpha}(\omega)=-\operatorname{Im} \Delta_{\alpha}^{\mathrm{hyb}}(\omega)$ describes the single-particle broadening of individual impurity levels $\alpha$ due to the coupling to the conduction electrons. Note that we have assumed here that each impurity level $\alpha$ couples to different conduction electron states (labeled by $\alpha$ ) in the substrate so that each impurity level has its own bath. This assumption is justified because of the different symmetries of the $d$-orbitals. In the case of coupling of two orbitals to the same conduction electron states, off-diagonal elements in the hybridization function $\Delta_{\alpha \alpha^{\prime}}^{\text {hyb }}(\omega)$ would occur, describing substrate mediated hopping between impurity levels. In adatom-substrate systems these off-diagonal elements are often either zero or very small $[30,31]$.

We now solve the Anderson model (2) within the socalled One-Crossing Approximation (OCA) [32-34]. The first step is an exact diagonalization of the isolated impurity Hamiltonian (3):

$$
\mathcal{H}_{\mathrm{imp}}=\sum_{m} E_{m}|m\rangle\langle m|
$$

The many-body eigenstates $|m\rangle$ are eigenstates of the total number of electrons of the impurity, i.e. $\hat{N}_{d}|m\rangle=N_{m}|m\rangle$, and the total spin $S^{2}$ of the impurity, i.e. $\hat{S}^{2}|m\rangle=$ $S_{m}\left(S_{m}+1\right)|m\rangle$. For a ground state (GS) with integer spin $S$, and magnetic anisotropy with negative uniaxial anisotropy $D \neq 0$ and finite in-plane anisotropy $E \neq 0$, the $(2 S+1)$ degeneracy of the GS multiplet is completely lifted. This is schematically shown in Figures $1 \mathrm{~b}, 1 \mathrm{c}$ for $S=1$ and $S=2$ and $D<0[2,4]$ : for $D<0$ and $E=0$ the GS is doubly degenerate with the GS doublet having the maximal spin projection $m_{z}= \pm S$. A finite in-plane anisotropy $E$ allows for quantum tunneling between both spin directions thus lifting the degeneracy of the GS doublet, which now becomes split by $\Delta_{0}$, the bare quantum spin tunneling. The quantum states of the split doublet are thus linear combinations $| \pm\rangle \sim\left|m_{z}=+S\right\rangle \pm\left|m_{z}=-S\right\rangle$.
In the next step a diagrammatic expansion of the many-body eigenstates $|m\rangle$ of the (isolated) impurity $\mathcal{H}_{\text {imp }}$ in terms of the hybridization (5) is developed. $\mathcal{V}_{\text {hyb }}$ connects eigenstates $|m\rangle$ and $|n\rangle$ of $\mathcal{H}_{\text {imp }}$ with occupation numbers differing by one $\left(N_{m}=N_{n} \pm 1\right.$, see (A.1) in the Appendix). It is these fluctuations between the impurity GS and excited states with one more or one less electron that give rise to the Kondo effect. To this end one introduces so-called pseudo-particles (PPs) $m$ corresponding to the many-body eigenstates $|m\rangle$. The full propagator of such a PP $m$ can be written as:

$$
G_{m}(\omega)=\frac{1}{\omega-\lambda-E_{m}-\Sigma_{m}(\omega)},
$$

where $\Sigma_{m}(\omega)$ is the PP self-energy which describes the renormalization (real part) and broadening (imaginary part) of the PP $m$ due to the interaction with other PPs $m^{\prime}$ mediated by the conduction electron bath (see Eq. (A.11) in Appendix). $-\lambda$ is the chemical potential for the PPs which has to be adjusted such that the total PP charge is constrained to one (see Eqs. (A.4) and (A.6) in the Appendix).

OCA consists in a diagrammatic expansion of the PP self-energies $\Sigma_{m}$ in terms of the hybridization function $\Delta_{\alpha}^{\text {hyb }}$ to infinite order but summing only a subset of diagrams (only those involving conduction electron lines crossing at most once). This leads to a set of coupled integral equations for the PP propagators and selfenergies that have to be solved self-consistently. Once the OCA equations are solved the real electron spectral function $\rho_{\alpha}(\omega)$ for the impurity levels entering equation (1) for calculating the conductance spectrum is obtained from convolutions of the $\mathrm{PP}$ spectral functions $A_{m}(\omega)=-\operatorname{Im} G_{m}(\omega) / \pi$ (see Eqs. (A.16)-(A.17) in Appendix) which feature sharp resonances at the renormalized many-body energies $E_{m}^{*}=E_{m}+\operatorname{Re} \Sigma_{m}\left(E_{m}^{*}\right)$. The differences between the renormalized energies of the excited states $E_{m}^{*}$ and the GS $E_{0}^{*}$ yield the real electronic excitations as shown in Figure 1d.

OCA captures both the weak and strong coupling Kondo regimes, and has shown to produce reliable spectra for the single-orbital Anderson model, as long as the temperatures are not too low compared to the Kondo temperature $[35,36]$. For the general multi-orbital situation considered here, benchmarking of spectra is difficult since Numerical Renormalization Group [37] is computationally too demanding to be applied. Using Continuous-Time Quantum Monte-Carlo, it has been shown [38] that OCA is markedly superior to the simpler Non-Crossing Approximation (NCA) for dealing with multi-orbital Anderson models, although certain sum rule violations are found. Very importantly, OCA has shown excellent agreement with experiments in very complex multi-orbital systems $[17,39,40]$. More details about the OCA method are given in the Appendix. 

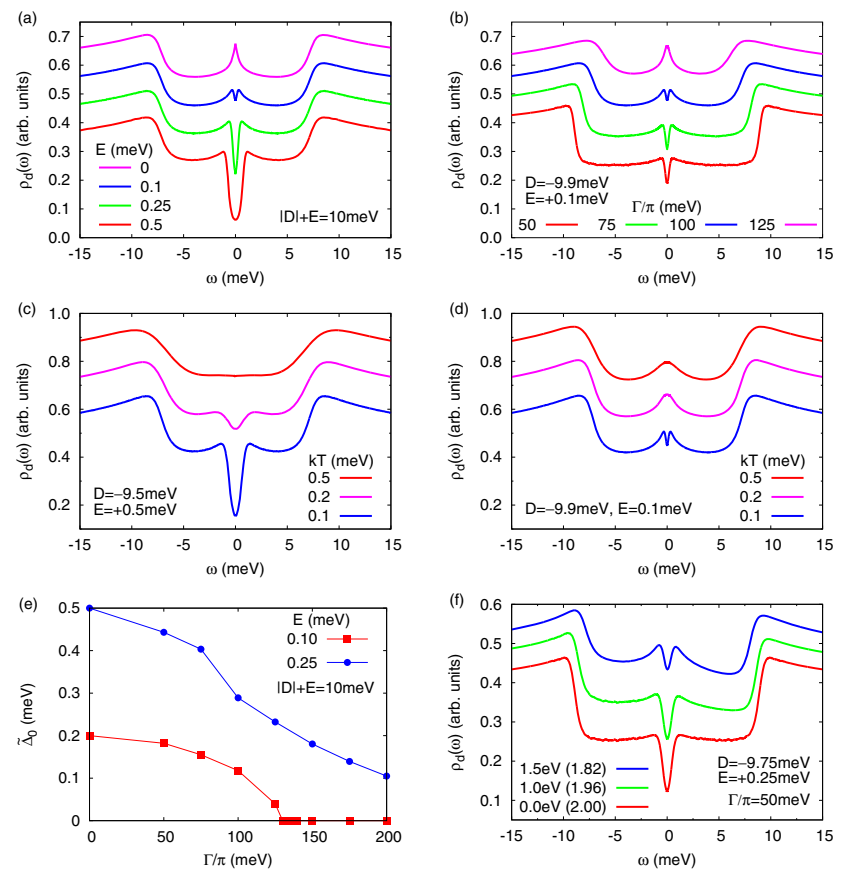

Fig. 2. Results for spin $S=1$ and for $D<0$, and temperature $k T=0.1 \mathrm{meV}$. (a)-(d) Spectra $\rho_{d}(\omega)$ in dependence of different system parameters: (a) changing $E$ while keeping fixed $\Delta_{1}=|D|+E=10 \mathrm{meV}$ and $\Gamma / \pi=100 \mathrm{meV}$. (b) Changing $\Gamma$ for $D=-9.9 \mathrm{meV}$ and $E=0.1 \mathrm{meV}$. (c) Temperature dependence for $\Gamma / \pi=100 \mathrm{meV}, D=-9.75 \mathrm{meV}$ and $E=0.25 \mathrm{meV}$. (d) Same as (c) but for $D=-9.9 \mathrm{meV}$ and $E=0.1 \mathrm{meV}$. (e) Effective QST $\tilde{\Delta}_{0}$ as a function of $\Gamma$. (f) Spectra for $D=-9.75 \mathrm{meV}, E=0.25 \mathrm{meV}$ and $\Gamma / \pi=100 \mathrm{meV}$ for different energy level shifts $\delta \epsilon_{d}$. In parenthesis the total occupancy $N_{d}$ of the impurity is given.

\section{Results}

We assume that the spin $S$ of the impurity is carried by $2 S$ active impurity levels close to half-filling. For all impurity levels we assume the same energy-independent broadening $\Gamma$. The important energy scale is the Kondo exchange coupling $J \sim \Gamma / \mathcal{U}$ which can be varied by either changing $\Gamma$ or the charging energy $\mathcal{U}$ which depends on the Coulomb repulsion $U$. Here we choose to vary $\Gamma$ and fix the Coulomb repulsion to $U=4 \mathrm{eV}$. The Hund's coupling whose main effect is to favor the formation of a high-spin ground state is fixed to $J_{\mathrm{H}}=1 \mathrm{eV}$.

We first study the competition between Kondo quenching and QST for a magnetic atom with spin $S=1$, at the point of electron-hole (e-h) symmetry. In this case the competition is controlled by two energy scales, the in-plane anisotropy $E$, that drives the QST, and $\Gamma$, that favors Kondo coupling. We assume $D<0$ so that, for $E=0$, the ground state is the doublet of eigenstates of $S_{z}$ with $m_{z}= \pm 1$ and the state with $m_{z}=0$ is the first excited state (see Fig. 1b). Because of the two-fold degeneracy of the GS the Kondo effect can take place (top curve in Fig. 2a). Spin-flip events for the GS doublet $m= \pm 1$ occur via the excited state $m=0$ and hence are reduced by a factor of $J / D$ compared to the Kondo exchange $J$ of the corresponding spin-1 Kondo model without anisotropy. Note that such a spin-flip process involves the simultaneous scattering of two conduction electrons. This is possible since we are considering a multichannel situation as each impurity orbital is connected to its own bath. In the situation of just a single screening channel, the spin-flip between the $m= \pm 1$ GS doublet would be inhibited when $D$ becomes bigger than the Kondo temperature, leading to a split-Kondo feature [41,42].

The in-plane anisotropy term $E>0$ produces the QST that leads to a splitting of the $m= \pm 1$ doublet, so that the bare excitation energies are $\Delta_{0}=2 E$ and $\Delta_{1}=|D|+E$. At weak coupling (small $\Gamma$ ), the spectra show two steps corresponding to inelastic spin transitions between the renormalized spin levels (bottom curve in Fig. 2a). Our calculations show that the effect of decreasing $E$ (Fig. 2a) and increasing $\Gamma$ (Fig. 2b) is similar. In both instances the renormalized QST splitting $\tilde{\Delta}_{0}$ decreases, and the line shapes evolve from square steps at small $\Gamma$ or large $E$ to a characteristic triangular shape, very often seen in experiments $[4,5,19]$, that can only be captured in part when going beyond second order perturbation theory in the Kondo exchange $[24,25]$. Whereas the reduction of the renormalized QST splitting $\tilde{\Delta}_{0}$ as $E$ decreases is trivially accounted for by the fact that $\Delta_{0}=2 E$, the red-shift renormalization of the spin excitation energies due to Kondo coupling (Fig. 2e) is a many-body effect, in line with previous results $[16-18,43,44]$. In our approach the renormalization of the spin excitations ultimately originates in the renormalization of the many-body energies $E_{m}^{*}$ by the real part of the PP self-energy $\Sigma_{m}(\omega)$ (see Eq. (8)). We would like to stress at this point that the splitting of the Kondo peak is exactly given by the effective QST $\Delta_{0}$. Hence Figure 2e really is a prediction for the splitting of the Kondo peak by QST which can be measured experimentally.

At intermediate coupling, the renormalization of the line shape and energy of the lowest energy spin excitation turns them into a split Kondo peak. This is one of the important results of this work: in the absence of a magnetic field the competition between QST and Kondo effect yields a split-Kondo state whose characteristic signature can be measured by STM spectroscopy. As shown in Figures 2c and 2d, the temperature dependence of the spectra in the case of intermediate coupling (split-Kondo) is quite different from that of weak coupling (step-like). For weak coupling (Fig. 2c), at low temperatures two clear steps are obtained, with slight triangular departures from the step-wise behavior, due to Kondo interactions, that are captured as well by perturbative calculations [25]. As the temperature is increased, the low energy step is smeared out and, eventually, is no longer resolved. On the other hand, at intermediate coupling (Fig. 2d), a zerofield split Kondo peak is obtained at low temperatures. As we increase $k_{B} T$ the QST splitting of the Kondo peak disappears due to thermal smearing, resulting in a single peak. At strong coupling, the effective QST $\tilde{\Delta}_{0}$ vanishes (Fig. 2e), so that the ground state and the first excited state become effectively degenerate: Kondo coupling 

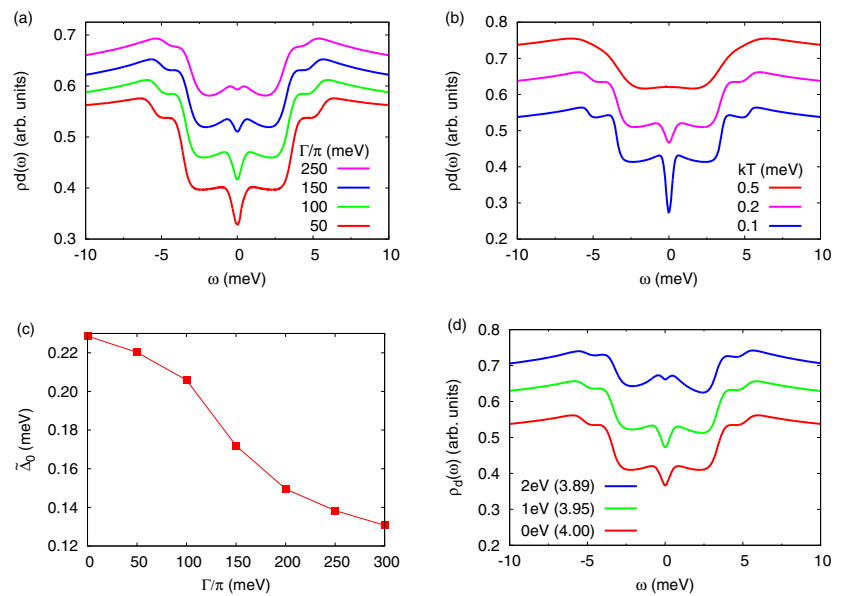

Fig. 3. Results for spin $S=2$ with $D=-1.55 \mathrm{meV}$ and $E=0.35 \mathrm{meV}$, and temperature $k T=0.2 \mathrm{meV}$. (a) Spectral function $\rho_{d}(\omega)$ for different values of single-particle broadening $\Gamma$. (b) Temperature dependence of spectral function for $\Gamma / \pi=100 \mathrm{meV}$. (c) QST $\tilde{\Delta}_{0}$ as a function of single-particle broadening $\Gamma$. (d) Effect of charge fluctuations: spectra for $\Gamma / \pi=100 \mathrm{meV}$ for different energy level shifts $\delta \epsilon_{d}$. In parenthesis the total occupancy $N_{d}$ of the impurity levels is given.

quenches QST splitting [16]. At this point, the spectra show a Kondo peak (top curves in Figs. 2a and 2b).

We now consider the effect of valence fluctuations. This effect is particularly important since DFT calculations show that often the average occupation of the $d$ shell is not quantized $[26,27]$. In our model, we control the valence mixing by shifting the impurity levels by an amount $\delta \epsilon_{d}$, taking the system out of the e-h symmetry point, leading to deviations from integer occupation number of the impurity shell. Valence fluctuations have a similar effect on the spectra as increasing the Kondo screening, leading e.g. to a considerable enhancement of the Kondo peak [45]. Similarly, also the renormalization of the excitation energies and especially of the QST by the Kondo is enhanced by the introduction of charge fluctuations, as can be seen in Figure 2f. Increasing $\delta \epsilon_{d}$ leads to the step features associated with spin excitations moving to lower energies. Also the up-bending of the lowest energy steps associated with QST and the conversion into a split-Kondo peak is induced by increasing valence fluctuations. Different from the case when $\Gamma$ increases, the spectra also become somewhat asymmetric as the e-h symmetry is broken by the valence fluctuations.

The competition between Kondo effect and QST is also present for higher integer spin systems. In Figure 3 we show results for spin $S=2$, and magnetic anisotropy parameters $D=-1.55 \mathrm{meV}$ and $E=0.35 \mathrm{meV}$ chosen to reproduce spectra for $\mathrm{Fe}$ on $\mathrm{Cu}_{2} \mathrm{~N}$ [4]. The energy level diagram, and the spin composition of the corresponding states, are shown in Figure 1. Here the QST splits both the $m= \pm 2$ ground state doublet, by an amount $\Delta_{0} \propto \frac{E^{2}}{D}$ as well as the $m= \pm 1$ doublet by $2 E$. Thus, the magnetic anisotropy completely lifts the degeneracy of the GS quintuplet leading to step features in the calculated spectral functions (Fig. 3a) that, for small values of $\Gamma$, resemble those measured for $\mathrm{Fe}$ on $\mathrm{Cu}_{2} \mathrm{~N}$ [4], and correspond to inelastic spin excitations from the ground state. As $\Gamma$ is increased, the inelastic spin excitation step features both move to lower energies and become broader, indicating both the renormalization of the associated excitation energies and the decrease of the lifetimes due to the exchange coupling. As in the $S=1$ case, the step features associated with QST develop a triangular shape with increasing $\Gamma$ and finally turn into a split-Kondo peak. As $\Gamma$ is increased, the effective QST $\tilde{\Delta}_{0}$ is renormalized but remains finite in the range of physically reasonable values of $\Gamma$ (up to $1 \mathrm{eV}$ ) considered here (see Fig. 3c). We note that generally the Kondo coupling is also weaker for $S=2$ than for $S=1$ since higher order processes are necessary to screen the spin. For a somewhat smaller $E$ (not shown) the splitting of the Kondo peak can also vanish for spin $S=2$. Figure $3 \mathrm{~b}$ shows the temperature dependence of the spectrum for the case of relatively small coupling $\Gamma$. As the temperature is lowered, initially flat steps appear in the spectrum, which become increasingly triangular as the temperature is lowered further. As in the case of spin $S=1$, increasing the valence fluctuations by detuning the system from ph symmetry has a similar effect as increasing $\Gamma$ (see Fig. 3d), leading to a red-shift of the spin excitation energies. The detuning from ph symmetry also leads to an up-bending of the initially flat step features associated with QST and to the conversion into a splitKondo peak.

The picture that emerges from our calculations is the following. An atom with integer spin $S$, described with Hamiltonian (3), is a closed quantum system whose quantum ground state has a built-in coherence between the two classical ground states, with $S_{z}= \pm S$, associated to the QST splitting $\Delta_{0}$, that would determine the frequency of the Rabi flops of the magnetization if the atom was initially prepared in an eigenstate of $S_{z}$. As the coupling to the surface electrons is turned on, the atomic spin behaves like a quantum open system. This results both in the renormalization (reduction) of this Rabi frequency as well as spin relaxation, leading to a broadening of the steps. For sufficiently large coupling the QST splitting can be completely quenched, in line with previous results [16], and quantum coherence between the states with opposite $S_{z}$ is lost. At that point Kondo screening takes over, and the zero bias Kondo feature appears.

Interestingly, the evolution between the strong coupling Kondo regime and the weak coupling with step-wise excitations is continuous. At intermediate couplings the spectral functions show a split Kondo peak, that resembles the Zeeman split Kondo peak, but is driven by QST instead. The strength of the Kondo coupling is controlled both by $\Gamma$ and by the departure from the e-h symmetry point, that we change by tuning $\delta \epsilon_{d}$. Importantly, both the weak coupling picture with step-wise excitations associated with spin transitions, and the strong coupling Kondo regime, are also obtained in the case when the charge on the atom is not quantized, as suggested by DFT calculations $[26,27]$. 


\section{Conclusions}

In summary, we have studied the competition between two important physical phenomena that affect integer spins, namely QST and Kondo screening. Our calculations permit to trace the evolution from the weak Kondo coupling regime, where the stepwise $d I / d V$ spectra are renormalized, resulting in the shift and the broadening of the spin excitation energies, to the strong coupling regime, where the zero bias Kondo peak appears. This accounts for several experimental observations [17,20]. For strong Kondo coupling, QST can be completely quenched. Importantly, for the intermediate coupling regime we predict a new physical effect: for $B=0$ an energy split Kondo peak can arise, because of quantum spin tunneling splitting, in analogy with the $B \neq 0$ Zeeman split Kondo peak, recently investigated more closely by spin-polarized STM [46].

\section{Author contribution statement}

JFR proposed to study the effect of Kondo screening on quantum spin tunneling in integer spin systems. DJ implemented the solution of the Anderson model including the anisotropy term within the OCA code and performed all numerical calculations. Both authors contributed equally to the discussion and physical interpretation of the results and to the writing of the manuscript.

JFR acknowledges financial support by MEC-Spain (FIS2013 47328 C2 2 P) and Generalitat Valenciana (ACOMP 2010 070), Prometeo. We thank L. Glazman, R. Žitko and C. Hirjibehedin for fruitful discussions during SPICE workshop "Magnetic adatoms as building blocks for quantum magnetism".

\section{Appendix: One-crossing approximation}

The starting point are the eigenstates $|m\rangle$ and corresponding eigenenergies $E_{m}$ of the isolated impurity Hamiltonian (7). In terms of the impurity eigenstates $|m\rangle$ we can rewrite the hybridization term $\mathcal{V}_{\text {hyb }}$ as:

$$
\mathcal{V}_{\text {hyb }}=\sum_{m, n} \sum_{k, \alpha, \sigma} V_{k \alpha}\left(|m\rangle\left\langle m\left|d_{\alpha \sigma}^{\dagger}\right| n\right\rangle\langle n| c_{k \alpha \sigma}+\text { h.c. }\right) \text {. }
$$

$\mathcal{V}_{\text {hyb }}$ connects eigenstates of $\mathcal{H}_{\text {imp }}$ with different occupation numbers, i.e. $N_{m}=N_{n} \pm 1$. It is the fluctuations between the GS and excited states of the atom give rise to the Kondo effect. Since the electron-electron interaction on the impurity $\left(U\right.$ and $J_{H}$ ) is generally large compared to the hybridization $\left(V_{k \alpha}\right)$, a perturbative treatment in terms of the latter is justified.

We are now going to develop a perturbation theory for the many-body eigenstates $|m\rangle$ of $\mathcal{H}_{\text {imp }}$ in terms of the hybridization term $\mathcal{V}_{\text {hyb }}$. In order to proceed we associate so-called pseudo-particle (PP) field operators $a_{m}^{\dagger}, a_{m}$ with the many-body eigenstates $|m\rangle$ of $\mathcal{H}_{\mathrm{imp}}$ :

$$
|m\rangle=a_{m}^{\dagger}|\tilde{0}\rangle \text { and } a_{m}|m\rangle=|\tilde{0}\rangle,
$$

where $|\tilde{0}\rangle$ is the PP vacuum. The auxiliary fields $a_{m}^{\dagger}$ and $a_{m}$ obey either commutation or anticommutation rules depending on whether the corresponding many-body state is bosonic (even number of electrons) or fermionic (odd number of electrons):

$$
\left[a_{m}, a_{m}^{\dagger}\right]=1 \text { (Bosons); }\left\{a_{m}, a_{m}^{\dagger}\right\}=1 \text { (Fermions). }
$$

Note that $a_{m}^{\dagger}$ and $a_{m}$ are only auxiliary fields that per se do not have a physical meaning. In order to give this construction a physical significance one has to impose an additional constraint that enforces the conservation of the PP number to one, i.e. the system can only be in one and only one state $|m\rangle$ at a time:

$$
Q=\sum_{m} a_{m}^{\dagger} a_{m} \equiv 1
$$

The PPs are related to the real electrons by:

$$
d_{\alpha \sigma}=\sum_{m, n}\left\langle m\left|d_{\alpha \sigma}\right| n\right\rangle a_{m}^{\dagger} a_{n}
$$

In the PP picture the Hamiltonian of the isolated atom becomes

$$
\mathcal{H}_{\mathrm{imp}}=\sum_{m} E_{m} a_{m}^{\dagger} a_{m}+\lambda\left(\sum_{m} a_{m}^{\dagger} a_{m}-1\right),
$$

where the last term is a Lagrangian constraint imposing the afore mentioned conservation of the PP charge $Q$. The Lagrange multiplier $\lambda$ can be seen as a (negative) chemical potential for the PPs. Imposing the constraint via the Lagrange multiplier is done e.g. in the Slave-Boson MeanField Approximation of the Anderson model [47]. However, for the diagrammatic expansion it is more convenient to work in the grand-canonical ensemble with respect to the PP charge $Q$. The expectation values of a physical observable $A$ can be calculated in the grand canonical PP ensemble and then projected to the physical subspace $(Q=1)$ via the Abrikosov trick [48]:

$$
\langle A\rangle=\lim _{\lambda \rightarrow \infty} \frac{\langle Q A\rangle_{\lambda}}{\langle Q\rangle_{\lambda}} .
$$

In the PP picture the hybridization term (A.1) becomes:

$$
\mathcal{V}_{\mathrm{hyb}}=\sum_{m, n} \sum_{k, \alpha, \sigma} V_{k \alpha}\left(D_{m n}^{\alpha \sigma \dagger} a_{m}^{\dagger} a_{n} c_{k \alpha \sigma}+D_{m n}^{\alpha \sigma} c_{k \alpha \sigma}^{\dagger} a_{m}^{\dagger} a_{n}\right)
$$

where we have introduced the matrix elements $D_{m n}^{\alpha \sigma}=$ $\left\langle m\left|d_{\alpha \sigma}\right| n\right\rangle$ and $D_{m n}^{\alpha \sigma \dagger}=\left\langle m\left|d_{\alpha \sigma}^{\dagger}\right| n\right\rangle$. The hybridization term $\mathcal{V}_{\text {hyb }}$ is now treated as a perturbation to the Hamiltonian of the uncoupled impurity and bath $\mathcal{H}_{0}=$ $\mathcal{H}_{\text {imp }}+\mathcal{H}_{\text {bath }}$. The introduction of PPs obeying (anti-) commutation relations allows us to make use of the machinery of quantum field theory to develop a diagrammatic perturbation expansion since Wick's theorem applies.

We now introduce the bare PP propagators $G_{m}^{0}(\tau)=$ $-\left\langle T_{\tau} a_{m}(\tau) a_{m}^{\dagger}(0)\right\rangle_{0}$ which will be denoted by dashed lines:

$$
G_{m}^{0}(i \omega)=\frac{1}{i \omega-\lambda-E_{m}}=--\underset{m}{-}-.
$$


The bath electron propagator is denoted by a full line:

$$
g_{k \alpha \sigma}(i \omega)=\frac{1}{i \omega-\epsilon_{k \alpha}}=\underset{k \alpha \sigma}{\longrightarrow} .
$$

Expanding the full $\mathrm{PP}$ propagator $G_{m}(\tau)=$ $\left\langle T_{\tau} a_{m}(\tau) a_{m}^{\dagger}(0)\right\rangle$ in terms of the hybridization $\mathcal{V}_{\text {hyb }}$, we can integrate out the bath electrons, and end up with an effective retarded two-particle interaction between PPs mediated by the bath electrons:

$$
\begin{aligned}
\tilde{\mathcal{V}}_{\mathrm{hyb}}\left(\tau-\tau^{\prime}\right)= & \sum_{\alpha, \sigma, m, n, m^{\prime}, n^{\prime}} D_{m n}^{\alpha \sigma \dagger} D_{n^{\prime} m^{\prime}}^{\alpha \sigma} \\
& \times a_{m}^{\dagger}(\tau) a_{n}(\tau)\left[\sum_{k}\left|V_{k \alpha}\right|^{2} g_{k \alpha \sigma}\left(\tau-\tau^{\prime}\right)\right] \\
& \times a_{n^{\prime}}^{\dagger}\left(\tau^{\prime}\right) a_{m^{\prime}}\left(\tau^{\prime}\right) .
\end{aligned}
$$

The term in square brackets is called the hybridization function $\Delta_{\alpha}\left(\tau-\tau^{\prime}\right)$ which in the real frequency domain is given by equation (6).

The full PP propagators are denoted by double dashed lines. In terms of a PP self-energy $\Sigma_{m}(\omega)$ which captures the interaction with other PPs via $\mathcal{V}_{\text {hyb }}$, the full propagator can be written as:

$$
G_{m}(i \omega)=\frac{1}{i \omega-\lambda-E_{m}-\Sigma_{m}(i \omega)}==\mathbf{m}=\mathbf{=} .
$$

In lowest order (2nd order in $V_{k \alpha}$ or 1st order $\Delta_{\alpha}$ ) the PP self-energy is given by the following diagrams:

$$
\Sigma_{m}(\omega)=\overbrace{-\rightarrow m^{\prime}}^{\alpha \sigma}+\overbrace{-\rightarrow m^{\prime}}^{\alpha \sigma} .
$$

The conduction electron line (full lines) $\alpha \sigma$ correspond to the hybridization function $\Delta_{\alpha}(\omega)$.

The first diagram ("backward" diagram) corresponds to adding an electron to the impurity site, i.e. $N_{m^{\prime}}=$ $N_{m}+1$ and the second diagram ("forward" diagram) to removing an electron, i.e. $N_{m^{\prime \prime}}=N_{m}-1$. The non-crossing approximation (NCA) consists in an infinite resummation of these lowest order diagrams where conduction electron lines do not cross (hence the name) [49]. Replacing the bare propagators by full propagators in the above self-energy diagrams, one obtains the NCA self-energy diagrams:

$$
\begin{aligned}
& \Sigma_{m}^{\mathrm{NCA}}(\omega)=\delta=\overbrace{m^{\prime}}^{\alpha \sigma}=\boldsymbol{\phi}+\overbrace{=\underset{m^{\prime}}{=}=\boldsymbol{b}}^{\alpha \sigma} \\
& =-\sum_{m^{\prime} \alpha \sigma} \int \frac{d \nu}{\pi}\left[\left|D_{m m^{\prime}}^{\alpha \sigma}\right|^{2} f(\nu) \Gamma_{\alpha}(\nu) G_{m^{\prime}}(\omega+\nu)\right. \\
& \left.+\left|D_{m^{\prime} m}^{\alpha \sigma}\right|^{2} f(-\nu) \Gamma_{\alpha}(\nu) G_{m^{\prime}}(\omega-\nu)\right]
\end{aligned}
$$

where $\Gamma_{\alpha}(\omega) \equiv-\operatorname{Im} \Delta_{\alpha}(\omega)$ is the single-particle broadening of the impurity levels $\alpha$ by the coupling to the conduction electron bath, and $f(\nu)$ is the Fermi function. Hence the NCA self-energy for a PP $m$ is given by a convolution of the imaginary part of the hybridization function $\Gamma_{\alpha}$ with the propagators of all other PPs $m^{\prime}$ that $m$ is interacting with via the conduction electron bath.

The OCA diagrams are second order in $\Delta_{\alpha}(\omega)$ and involve crossing conduction electron lines:

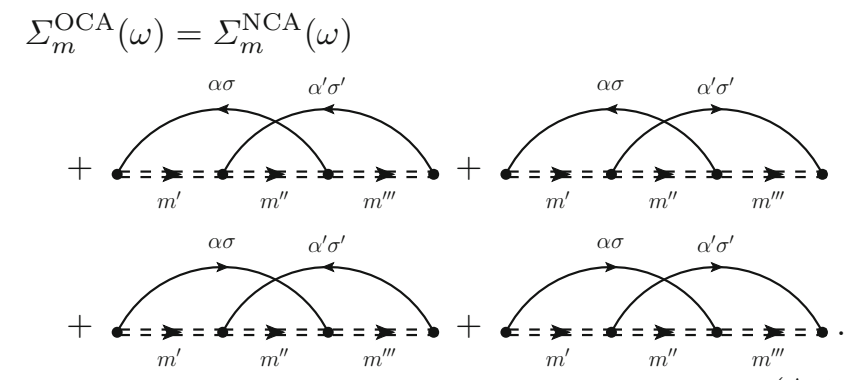

(A.15)

The algebraic expressions for the OCA self-energy are much more complicated than the NCA ones, and involve double convolutions of two hybridization functions with three PP propagators. The exact expressions can be found in references [33,34].

The NCA/OCA equations are a set of coupled integral equations that have to be solved self-consistently since the self-energy of a PP $m$ depends on the full propagators of other PPs $m^{\prime}$. Once the NCA/OCA equations are solved, the real impurity electron propagator can be determined by making use of the relation (A.5). Hence the real electron propagator can be calculated from a two-particle correlation function for PPs:

$$
\begin{aligned}
G_{\alpha \sigma}(\tau) & =-\left\langle T_{\tau} d_{\alpha \sigma}(\tau) d_{\alpha \sigma}^{\dagger}(0)\right\rangle \\
& =-\sum_{m, n, m^{\prime}, n^{\prime}} D_{m n}^{\alpha \sigma \dagger} D_{n m}^{\alpha \sigma}\left\langle T_{\tau} a_{m}^{\dagger}(\tau) a_{n}(\tau) a_{n}^{\dagger} a_{m}\right\rangle
\end{aligned}
$$

In NCA the vertex correction $\Lambda$ is neglected. It is then found that the real electron spectral function $\rho_{\alpha \sigma}(\omega)=$ $-\operatorname{Im} G_{\alpha \sigma}(\omega) / \pi$ can be calculated from a convolution of PP spectral functions $A_{m}(\omega)=-\operatorname{Im} G_{m}(\omega) / \pi$ :

$$
\begin{aligned}
\rho_{\alpha}(\omega)= & \frac{1}{\langle Q\rangle_{\lambda}} \sum_{m m^{\prime}} \int d \varepsilon e^{-\beta \varepsilon}\left[1+e^{-\beta \omega}\right] \\
& \times\left|D_{m m^{\prime}}^{\alpha \sigma}\right|^{2} A_{m}(\varepsilon) A_{m^{\prime}}(\omega+\varepsilon),
\end{aligned}
$$

where $Q$ is the PP charge which can be calculated directly from the PP spectral functions:

$$
\langle Q\rangle=\int d \omega e^{-\beta \omega} \sum_{m} A_{m}(\omega) .
$$

Again the corresponding expression for calculating the real electron spectral function within OCA is much more complicated as it involves double convolutions of PP correlation functions and the hybridization function [33,34]. 


\section{References}

1. D. Gatteschi, R. Sessoli, J. Villain, Molecular Nanomagnets (Oxford University Press, Oxford, 2006)

2. A. Abragam, B. Bleaney, Electron Paramagnetic Resonance of Transition Ions (Oxford University Press, Oxford, 2012)

3. A.C. Hewson, The Kondo Problem to Heavy Fermions (Cambridge University Press, Cambridge, 1997)

4. C. Hirjibehedin, C-Y Lin. A.F. Otte, M. Ternes, C.P. Lutz, B.A. Jones, A.J. Heinrich, Science 317, 1199 (2007)

5. A.A. Khajetoorians, B. Chilian, J. Wiebe, S. Schuwalow, F. Lechermann, R. Wiesendanger, Nature 467, 1084 (2010)

6. N. Tsukahara, K.I. Noto, M. Ohara, S. Shiraki, N. Takagi, Y. Takata, J. Miyawaki, M. Taguchi, A. Chainani, S. Shin, M. Kawai, Phys. Rev. Lett. 102, 167203 (2009)

7. S. Sasaki, S. De Franceschi, J.M. Elzerman, W.G. van der Wiel, M. Eto, S. Tarucha, L.P. Kouwenhoven, Nature 405, $764(2000)$

8. J.J. Parks, A.R. Champagne, T.A. Costi, W.W. Shum, A.N. Pasupathy, E. Neuscamman, S. Flores-Torres, P.S. Cornaglia, A.A. Aligia, C.A. Balseiro, G.K.-L. Chan, H.D. Abruña, D.C. Ralph, Science 328, 1370 (2010)

9. N. Tsukahara, S. Shiraki, S. Itou, N. Ohta, N. Takagi, M. Kawai, Phys. Rev. Lett. 106, 187201 (2011)

10. A. Mugarza, R. Robles, C. Krull, R. Korytar, N. Lorente, P. Gambardella, Phys. Rev. B 85, 155437 (2012)

11. Y. Jiang, Y.N. Zhang, J.X. Cao, R.Q. Wu, W. Ho, Science 333, 324 (2011)

12. V. Madhavan, W. Chen, T. Jamneala, M.F. Crommie, N.S. Wingreen, Science 280, 567 (1998)

13. A. Garg, Europhys. Lett. 22, 205 (1993)

14. R. Sessoli, W. Wernsdorfer, Science 284, 133 (1999)

15. F. Delgado, J. Fernández-Rossier, Phys. Rev. Lett. 108, $196602(2012)$

16. F. Delgado, S. Loth, M. Zielinski, J. Fernández-Rossier, EPL 109, 57001 (2015)

17. J. Oberg et al., Nat. Nanotech. 9, 64 (2014)

18. F. Delgado, C.F. Hirjibehedin, J. Fernández-Rossier, Surf. Sci. 630, 337 (2014)

19. A.F. Otte, M. Ternes, K. von Bergmann, S. Loth, H. Brune, C.P. Lutz, C.F. Hirjibehedin, A.J. Heinrich, Nat. Phys. 4, 847 (2008)

20. P. Jacobson, T. Herden, M. Muenks, G. Laskin, O. Brovko, V. Stepanyuk, M. Ternes, K. Kern, Nat. Comm. 6, 8536 (2015)

21. J. Fernández-Rossier, Phys. Rev. Lett. 102, 256802 (2009)

22. R. Žitko, Th. Pruschke, New. J. Phys. 12, 063040 (2010)

23. R. Žitko, R. Peters, Th. Pruschke, Phys. Rev. B 78, 224404 (2008)

24. A. Hurley, N. Baadji, S. Sanvito, Phys. Rev. B 84, 115435 (2011)

25. M. Ternes, New J. Phys. 17, 063016 (2015)
26. A. Ferrón, J.L. Lado, J. Fernández-Rossier, Phys. Rev. B 92174407 (2015)

27. S.K. Panda, I. Di Marco, O. Grånäs, O. Eriksson, J. Fransson, Phys. Rev. B 93, 140101 (2016)

28. S. Datta, Electronic Transport in Mesoscopic Systems (Cambridge University Press, Cambridge, 1995)

29. O. Újsághy, J. Kroha, L. Szunyogh, A. Zawadowski, Phys. Rev. Lett. 85, 2557 (2000)

30. B. Surer, M. Troyer, Ph. Werner, T. O. Wehling, A.M. Läuchli, A. Wilhelm, A.I. Lichtenstein, Phys. Rev. B 85, $085114(2012)$

31. D. Jacob, J. Phys.: Condens. Matter 27, 245606 (2015)

32. T. Pruschke, N. Grewe, Z. Phys. B 74, 439 (1989)

33. K. Haule, S. Kirchner, J. Kroha, P. Wölfle, Phys. Rev. B 64, 155111 (2001)

34. K. Haule, C.H. Yee, K. Kim, Phys. Rev. B 81, 195107 (2010)

35. T.A. Costi, J. Kroha, P. Wölfle, Phys. Rev. B 53, 1850 (1996)

36. N. Grewe, S. Schmitt, T. Jabben, F.B. Anders, J. Phys.: Condens. Matter 20, 365217 (2008)

37. R. Bulla, T.A. Costi, T. Pruschke, Rev. Mod. Phys. 80, $395(2008)$

38. A. Rüegg, E. Gull, G. A. Fiete, A. J. Millis, Phys. Rev. B 87, $075124(2013)$

39. D. Jacob, M. Soriano, J. .J. Palacios, Phys. Rev. B 88, 134417 (2013)

40. S. Karan, D. Jacob, M. Karolak, C. Hamann, Y. Wang, A. Weismann, A.I. Lichtenstein, R. Berndt, Phys. Rev. Lett. 115, 016802 (2015)

41. C. Romeike, M.R. Wegewijs, W. Hofstetter, H. Schoeller, Phys. Rev. Lett. 97, 206601 (2006)

42. A.S. Zyazin, J.W.G. van den Berg, E.A. Osorio, H.S.J. van der Zant, N.P. Konstantinidis, M. Leijnse, M.R. Wegewijs, F. May, W. Hofstetter, C. Danieli, A. Cornia, Nano Lett. 10, 3307 (2010)

43. R. Žitko, O. Bodensiek, Th. Pruschke, Phys. Rev. B 83, 054512 (2011)

44. R. Korytár, N. Lorente, J.-P. Gauyacq, Phys. Rev. B 85, $125434(2012)$

45. T.A. Costi, A.C. Hewson, V. Zlatić, J. Phys.: Condens. Matter 6, 2519 (1994)

46. K. von Bergmann, M. Ternes, S. Loth, C.P. Lutz, A.J. Heinrich, Phys. Rev. Lett. 114, 076601 (2015)

47. D.M. Newns, N. Read, J. Phys. C 16, 3273 (1983)

48. A.A. Abrikosov, Physica 2, 21 (1965)

49. P. Coleman, Phys. Rev. B 29, 3035 (1984)

Open Access This is an open access article distributed under the terms of the Creative Commons Attribution License (http://creativecommons.org/licenses/by/4.0), which permits unrestricted use, distribution, and reproduction in any medium, provided the original work is properly cited. 